\title{
Severe aortic stenosis with preserved ejection fraction and evidence of impairment in structure, myocardial strain and ventricular function: A new contribution to clinical decision making
}

\author{
Alejandro Hita ${ }^{1}$, Sergio Baratta ${ }^{1}$, Guillermo Vaccarino ${ }^{1}$, José Navia ${ }^{1}$, Daniel Olano ${ }^{1}$, \\ Juan Manuel Telayna ${ }^{1}$, Ricardo Costantini ${ }^{1}$, Demian Chejtman ${ }^{1}$, Miriam Matoso ${ }^{2}$, \\ Ricardo J. Gelpi ${ }^{2}$, Martin Donato ${ }^{2}$, Celina Morales ${ }^{2}$ \\ ${ }^{1}$ Cardiology Institute, Hospital Universitario Austral, Pilar, Buenos Aires, Argentina \\ ${ }^{2}$ Institute of Cardiovascular Pathophysiology, School of Medicine, \\ Universidad de Buenos Aires, Buenos Aires, Argentina
}

\begin{abstract}
Background: Left ventricular ejection fraction (LVEF) is among the parameters that are usually employed to define surgical timing of severe aortic stenosis (AS). Our hypothesis states that even when their LVEF is preserved, patients with severe symptomatic AS have impaired myocardial structure and function, and such impairment is related to the deleterious progression of left ventricular hypertrophy (LVH) from the compensated to the decompensated stage, as shown by the changes in diastolic function and the increase in left ventricular end-diastolic pressure (LVEDP).

Methods and Results: A total of 26 patients with severe AS and LVEF $>50 \%$ referred for aortic valve replacement underwent catheterization, echocardiography and an intraoperative biopsy. Patients with severe symptomatic AS were classified as: group 1 (G1; compensated LVH, $L V E D P<15 \mathrm{~mm} H g$ without coronary artery disease [CAD], $n=7$ ), group $2 a$ (G2a, decompensated $L V H$, without $C A D, n=7)$, and group $2 b$ (G2b, decompensated $L V H$ with $C A D$, $n=12)$. Differences were seen in the following: myocyte area $\left[\mu^{2}\right]: G 1: 328 \pm 66, G 2 a$ : $376 \pm 22$, G2b: $385 \pm 13, p<0.01$; collagen volume [\%]: G1: $4.77 \pm 1.27$, G2a: $8.40 \pm 1.27$, G2b: $11.05 \pm 3.08, p<0.01$; LVEDP normalized by diastolic diameter [mm Hg/mm]: G1: $0.27 \pm 0.01, G 2 a: 0.39 \pm 0.06, G 2 b: 0.44 \pm 0.11, p<0.02 ;+d P / d t_{\text {max }} / L V E D P[\mathrm{~mm} \mathrm{Hg} / \mathrm{s} / \mathrm{mm} \mathrm{Hg}]$ : G1: $176 \pm 45$, G2a: $89.6 \pm 20$, G2b: $113.1 \pm 41, p<0.01$; two-dimensional peak systolic longitudinal strain [\%]: G1: $-17.7 \pm 4.75$, G2a: $-13.4 \pm 3.04$, G2b: $-13.5 \pm 3.13, p<0.05$. Conclusions: Patients with severe symptomatic AS and preserved ejection fraction who develop decompensated LVH characterized by increased LVEDP, exhibit an abnormal myocardial structure and diastolic and systolic impairment. (Cardiol J 2015; 22, 6: 613-621)
\end{abstract}

Key words: aortic stenosis, hypertrophy, function

\section{Editorial p. 601}

Address for correspondence: Alejandro Hita, MD, Hospital Universitario Austral, Instituto de Cardiología, Av. Juan Domingo Perón 1500, B1629HJ, Derqui, Pilar, Buenos Aires, Argentina, tel: 54-11-47955299, e-mail: ahita@cas.austral.edu.ar 


\section{Introduction}

Management of patients with severe aortic stenosis and preserved ejection fraction (EF) is still controverted and the decision of whether to "watch and see" vs. early intervention remains debatable [1-3].

Current guidelines determine surgical timing according to symptoms and impairment of systolic function, defined by an EF below 50\% [4, 5]. Notwithstanding, some patients operated according to that criterion will have a poor outcome in the long-term follow-up, demonstrating that in fact, $\mathrm{EF}$ may not be sensitive enough to be used as a cutoff point and prognostic marker [6, 7].

The presence of fibrosis and myocyte changes together with an elevation in left ventricular (LV) end-diastolic pressure (LVEDP) or systolic function impairment reduce postoperative survival [8-11]. Hence, it could be useful to detect myocardial impairment in order to make therapeutic decisions before actual myocardial damage occurs. Along these lines, the use of new indices may help to identify such changes and define high risk populations that might benefit from an early surgical intervention [12].

Imaging with two-dimensional (2D) strain has been shown to be an appropriate method to assess myocardial contractility, as well as structure, and could be used to detect changes in LV performance $[13,14]$.

We hypothesize that in patients with severe symptomatic aortic stenosis (AS), even in the presence of a preserved EF, myocardial function and structure are impaired, and such impairment is related to the progression of LV hypertrophy ( $\mathrm{LVH})$, from the compensated to the decompensated stage, manifested by an increase in LVEDP.

In this study, we evaluated patients with severe symptomatic AS (SSAS) and preserved EF with the purpose of: a) detecting changes in LV systolic and diastolic function and their correlation with changes in myocardial structure, that could allow to discriminate different stages of progression of LVH, as assessed by LVEDP; b) analyzing 2D peak systolic longitudinal strain, preoperatively and 1 year after the aortic valve replacement, in agreement with de preoperative LVEDP values.

\section{Methods}

\section{Study population}

We prospectively included 26 consecutive patients (age: $67 \pm 11$ years, $53 \%$ male), with SSAS (defined by symptoms such as angina, syncope or dyspnea occurring at rest or with exercise) and by Doppler echocardiography (valve area $<1 \mathrm{~cm}^{2}$, mean gradient $>40 \mathrm{~mm} \mathrm{Hg}$ ) and an $\mathrm{EF}>50 \%$, referred to the Hospital Universitario Austral for aortic valve replacement. Prior to surgery, all patients underwent cardiac catheterization and Doppler echocardiography, and during valve replacement surgery a biopsy was obtained from the LV anterolateral wall. At 1 year post valve replacement, a new echocardiogram was performed. Patients with cardiomyopathy or other concomitant valve diseases were excluded. All patients accepted to participate in the study by signing an informed consent form (approved according to the Ethics Committee guidelines of the Hospital Universitario Austral) and the Declaration of Helsinki (1975). Patients with SSAS were divided into three groups according to their LVEDP $(<$ or $\geq 15 \mathrm{~mm} \mathrm{Hg}$ ) [15] and patients with a LVEDP $\geq 15 \mathrm{~mm} \mathrm{Hg}$ were further categorized according to the presence of coronary artery disease (CAD) (lesion in one coronary artery with obstruction $\geq 50 \%$ ): group 1 (G1; compensated LVH, LVEDP $<15 \mathrm{~mm} \mathrm{Hg}$ without CAD, $\mathrm{n}=7$ ), group 2a (G2a, decompensated $\mathrm{LVH}$ without $\mathrm{CAD}, \mathrm{n}=7$ ), group $2 \mathrm{~b}(\mathrm{G} 2 \mathrm{~b}$, decompensated LVH with CAD, $\mathrm{n}=12)$.

\section{Study protocol}

Echocardiography was performed with assessment of 2D peak systolic global longitudinal strain (SGLS) by speckle tracking technique, using Vingmed VIVID 7 equipment (GE Vingmed, Milwaukee, WT, USA) with a $3.5 \mathrm{MHz}$ transducer; measurements were performed according to recommendations of the American Society of Echocardiography (ASE) [16]. The following variables were calculated: LV fractional shortening, LV volume, EF (using Simpson's method) [16], and midwall fractional shortening (MFS) [17] which was normalized by end-systolic meridional stress [18]. Aortic valve area was calculated using the continuity equation and gradients with the modified Bernoulli equation $\left(4 \mathrm{~V}^{2}\right)$. Left ventricular mass (LVM) was calculated according to the ASE guidelines, and normalized by body surface to obtain the LVM index (LVMI); values were considered high when they exceeded $115 \mathrm{~g} / \mathrm{m}^{2}$ in men and $95 \mathrm{~g} / \mathrm{m}^{2}$ in women [19].

Assessment of $2 \mathrm{D}$ peak SGLS (\%) of the LV was performed with $2 \mathrm{D}$ echocardiography and grey scale speckle tracking imaging, using 2-, 3-, and 4-chamber views from the apical long axis, and a frame rate $>50$ frames/s [20]. The Lagrange formula $\left(\mathrm{L}_{2} \mathrm{~L}_{0} / \mathrm{L}_{0}\right)$ was used to calculate percent strain [21]. Strain was 
quantified using GE software (EchoPAC, version 7.0.0, General Electric Vingmed). The endocardial contour was traced manually at end-systole; subsequently the software automatically traced the region of interest concentrically [22]. For strain analysis, each apical view was divided into 6 segments and SGLS was then calculated from the average of 18 segments.

\section{Catheterization}

A LV catheterization was performed prior to surgery to record $\mathrm{LV}$ pressure and the aortic/LV gradient, which were recorded with a computerassisted system (Philips XPER (XIM) Xper Information System (XIMs) Polygraph, version 1.2.0.1474), connected to an Edwards Life Science pressure transducer. The following pressures were measured: aortic pressure, LV systolic pressure $[\mathrm{mm} \mathrm{Hg}]$ and LVEDP [mm $\mathrm{Hg}]$; the following indices were also calculated: maximal rate of rise in $\mathrm{LV}$ pressure $\left(+\mathrm{dP} / \mathrm{dt}_{\max }[\mathrm{mm} \mathrm{Hg} / \mathrm{s}]\right)$, the inverse slope of the line which results from applying the Ln to the equation of exponential drop in LV pressure during the isovolumic relaxation period (linear tau [ms]), and the time elapsed since $\mathrm{LV}$ pressure drops to $50 \%$ of its initial value $\left(t_{50}\right)$, considering as initial LV pressure the value corresponding to $-\mathrm{dP} / \mathrm{dt}_{\max }$.

The contractility index was calculated as the ratio between maximal rate of rise in $\mathrm{LV}$ pressure $\left(+\mathrm{dP} / \mathrm{dt}_{\max }[\mathrm{mm} \mathrm{Hg} / \mathrm{s}]\right)$ normalized by LVEDP [23].

The two components of diastolic function were evaluated: myocardial relaxation and myocardial chamber stiffness. To evaluate myocardial chamber stiffness we calculated the ratio between LV pressure and LV end-diastolic diameter. In order to assess the catheter sensitivity, the recording of ventricular pressure of the conventional catheter was compared in 3 patients with that obtained by a catheter tip transducer (Millar Instruments Inc.) that prevents potential problems caused by the fluid filled catheter. We observed that both the rise and decay slopes of the ventricular pressure were similar, therefore no differences were found in the measurements of the relaxation variables. As expected, measurements of the systolic and diastolic pressures were similar with both catheters.

\section{Histomorphometric analysis}

Myocardial biopsies of the LV free wall were obtained during aortic valve replacement procedures. Tissue samples were fixed in $10 \%$ buffered formalin at room temperature and paraffinembedded; subsequently, $5 \mu \mathrm{m}$ serial sections were obtained and stained with hematoxylin and eosin, Picrosirius red and rhodamine-conjugated lectin (WGA\# RL-1022, Vector Laboratories, Burlingame, CA).

Myocyte cross-sectional area was determined on digitized images of rhodamine-conjugated lectin-staining; such images were obtained using a fluorescence microscope (Olympus BX61) attached to a digital camera and connected to a computer equipped with image analysis software. Myocytes' outlines were traced and cell areas measured with Image-Pro Plus 6.0 (Media Cybernetics, Inc., Silver Spring, Md). At least 80 measurable cross sections of myocytes were routinely measured [24]. In the slices stained with Picrosirius red, interstitial collagen deposition was also measured using the image analysis system described above. Percent collagen volume for each region was calculated by adding the collagen areas and dividing that number by the sum of myocyte areas plus collagen areas, as previously described [24].

\section{Statistical analysis}

Parameter reproducibility was analyzed twice by the same observer (intraobserver variability) and by 2 independent operators in 8 patients (interobserver variability); variability coefficients were $5.4 \%$ and $6.2 \%$, respectively.

Qualitative variables were expressed as absolute values, proportions and/or percentages, with their 95\% confidence intervals (CI). Continuous variables were expressed as means and standard deviations, after accepting their normal distribution with D'Agostino-Pearson's test; when variables were not distributed normally, they were expressed as medians and inter-quartile ranges. Comparison among discrete variables was performed with Pearson's $\chi^{2}$ test, among continuous variables with the unpaired Student's t test for normal distributions and equal variances and for non-normal distributions with Mann-Whitney's $\mathrm{U}$ test. Association among continuous variables was determined with linear and nonlinear regression. To compare two or more independent groups, the one-way ANOVA test was used, with a post-hoc Tukey test to assess group differences. Tests were two-tailed and a $\mathrm{p}$ value $<0.05$ was considered statistically significant.

\section{Results}

The clinical characteristics of patients in the three groups $(\mathrm{n}=26)$ are depicted in Table 1 . 
Table 1. Clinical, hemodynamic and echocardiographic variables in basal condition.

\begin{tabular}{|c|c|c|c|c|}
\hline & Group 1 & Group 2a & Group 2b & $\mathbf{P}$ \\
\hline \multicolumn{5}{|l|}{ Clinical variables } \\
\hline Age & $68 \pm 12$ & $64.14 \pm 9.92$ & $71 \pm 11.59$ & NS \\
\hline Gender: male/female & $2 / 5$ & $3 / 4$ & $9 / 3$ & \\
\hline Systolic blood pressure [mm Hg] & $119.71 \pm 22.84$ & $125.14 \pm 5.87$ & $132 \pm 9.03$ & NS \\
\hline \multicolumn{5}{|l|}{ NYHA functional class for angina: } \\
\hline ॥ & 3 & 3 & 1 & \\
\hline III & 1 & & 2 & \\
\hline IV & & 1 & & \\
\hline \multicolumn{5}{|l|}{ NYHA functional class for dyspnea: } \\
\hline II & 3 & 6 & 4 & \\
\hline III & 3 & & 3 & \\
\hline \multicolumn{5}{|l|}{ IV } \\
\hline Syncope & 0 & 1 & 1 & NS \\
\hline \multicolumn{5}{|l|}{ Cardiac catheterization } \\
\hline LVESP [mm Hg] & $202.5 \pm 19.46$ & $211.28 \pm 40.47$ & $193.16 \pm 24.37$ & NS \\
\hline$+\mathrm{dP} / \mathrm{dt}_{\max }[\mathrm{mm} \mathrm{Hg} / \mathrm{s}]$ & $2,230.75 \pm 493.03$ & $1,830.28 \pm 346.98$ & $2,231.55 \pm 313.02$ & 0.07 \\
\hline $\operatorname{tau}_{50}$ & $24.99 \pm 2.41$ & $32.66 \pm 5.96$ & $32.52 \pm 8.85$ & NS \\
\hline \multicolumn{5}{|l|}{ Echocardiography } \\
\hline Ejection fraction [\%] & $75.71 \pm 5.93$ & $64 \pm 6.03$ & $66.72 \pm 10.65$ & 0.04 \\
\hline End-systolic diameter [mm] & $29.74 \pm 6.26$ & $26.33 \pm 5.39$ & $33.37 \pm 8.06$ & NS \\
\hline End-diastolic diameter [mm] & $49.31 \pm 5.29$ & $48.16 \pm 5.15$ & $52.75 \pm 4.15$ & NS \\
\hline Myocardial mass index $\left[\mathrm{g} / \mathrm{m}^{2}\right]$ & $198 \pm 85$ & $162 \pm 43$ & $209 \pm 42$ & NS \\
\hline $\begin{array}{l}\text { End-systolic meridional stress } \\
{\left[\mathrm{kdynes} / \mathrm{cm}^{2}\right]}\end{array}$ & $42.54 \pm 22.03$ & $37.7 \pm 14.98$ & $51.67 \pm 24.15$ & NS \\
\hline Midwall fractional shortening [\%] & $14.15 \pm 3.95$ & $14.96 \pm 2.7$ & $13.48 \pm 4.35$ & NS \\
\hline Midwall fractional shortening/stress & $18.68 \pm 0.83$ & $18.86 \pm 0.56$ & $18.05 \pm 1.04$ & NS \\
\hline Aortic valve area $\left[\mathrm{cm}^{2}\right]$ & $0.77 \pm 0.26$ & $0.45 \pm 0.07$ & $0.74 \pm 0.15$ & NS \\
\hline Mean aortic gradient [mm Hg] & $52.74 \pm 18.87$ & $66.83 \pm 10.83$ & $48.36 \pm 9.42$ & $<0.05$ \\
\hline Peak aortic gradient [mm Hg] & $89.28 \pm 26.32$ & $103.66 \pm 15.06$ & $80.09 \pm 16.1$ & 0.07 \\
\hline
\end{tabular}

1 - compensated hypertrophy; $2 \mathrm{a}$ - decompensated hypertrophy without coronary artery disease; $2 \mathrm{~b}$ - decompensated hypertrophy with coronary artery disease; LVESP — left ventricular end-systolic pressure; NS — not significant; NYHA — New York Heart Association

\section{Cardiac structure and function}

Endomyocardial biopsy. Differences among groups were seen in myocardial structure expressed by myocyte area $(\mathrm{p}<0.01)$ as well as by collagen volume $(\mathrm{p}<0.01)$ (Table 1$)$. The LVMI was similar in all groups; however, morphometric analysis showed that in group 1 myocyte area and collagen volume were smaller than in groups with decompensated LVH (Figs. 1A, B).

Patients in G1 exhibited a proportional increase in collagen volume for a certain increase in in myocyte area; such relation was lost in patients with decompensated $\mathrm{LVH}$, in whom for a certain increase in myocyte area there was a dispropor- tionate rise in collagen volume; this phenomenon reached its maximum expression in patients with CAD (Fig. 2).

Diastolic function. In the absence of changes in diastolic diameters, the difference in LVEDP between the three groups attests to an increase in myocardial stiffness in the groups with decompensated LVH (Fig. 3).

All patients had impairment in myocardial relaxation, expressed by a change in tau and tau $\mathrm{u}_{50}$, and without differences among groups (Table 1). The rise in LVEDP correlated directly with the changes in structure assessed by collagen volume (regression coefficient 0.97, p < 0.001) (Fig. 4). 


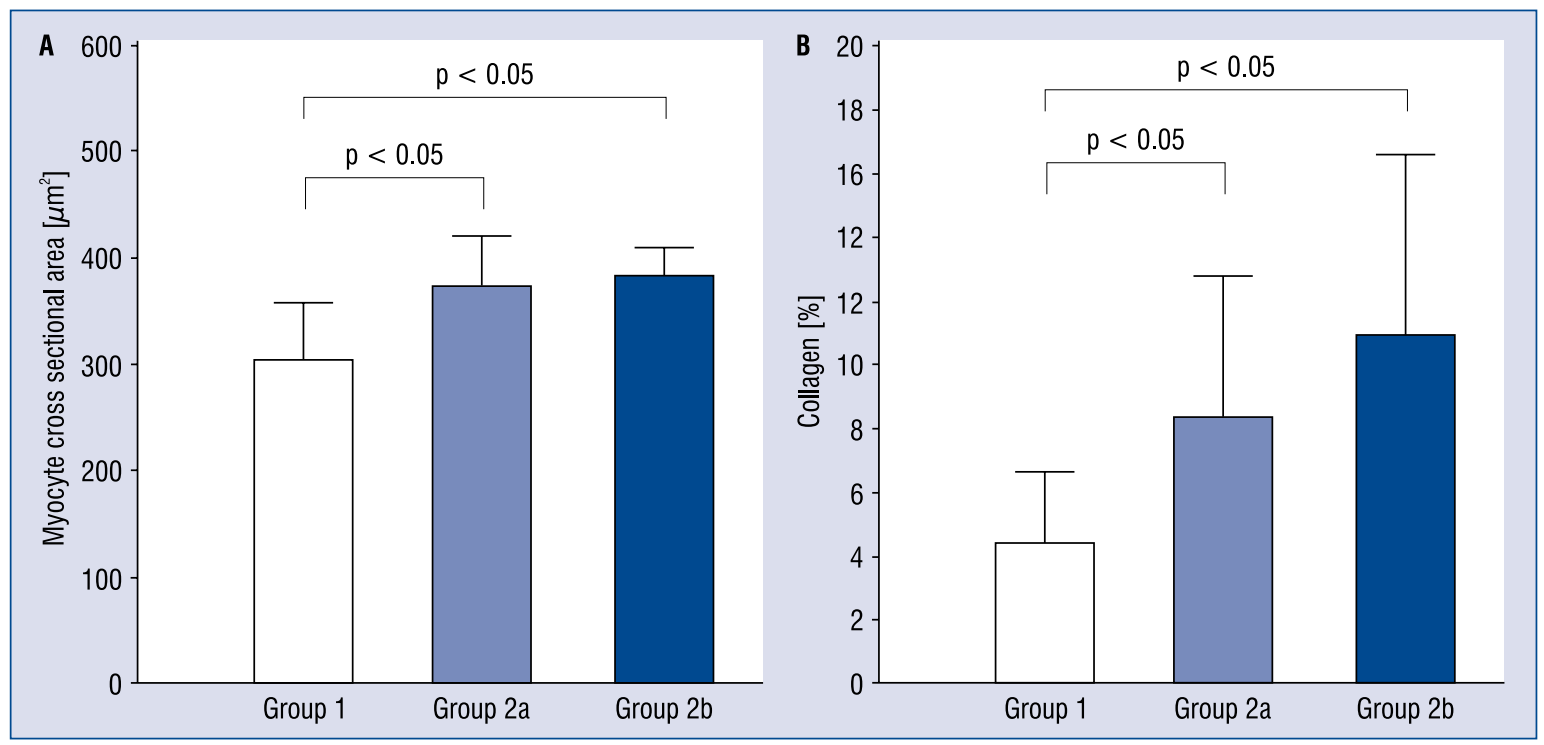

Figure 1. A. Differences in myocyte cross sectional area between groups; B. Differences in collagen volume expressed as percent between groups; * $p<0.05$ G1 vs. G2a and G2b.

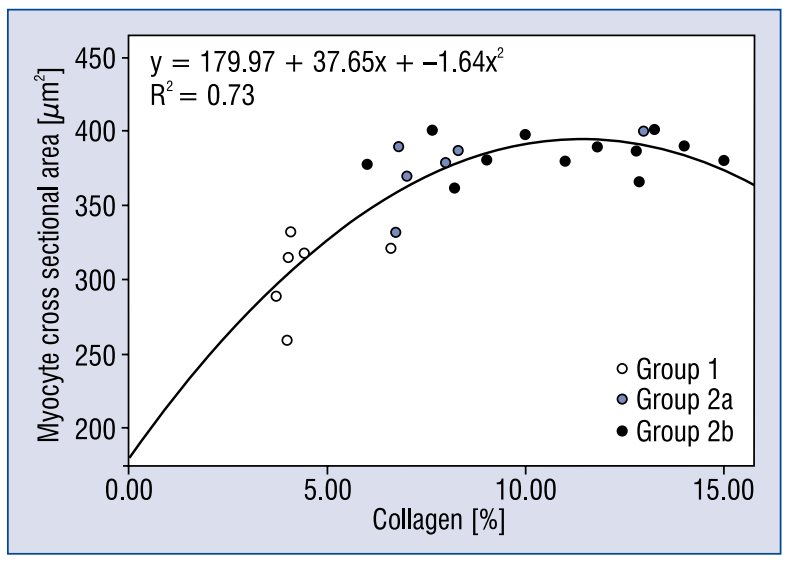

Figure 2. Quadratic function expressing the relation between collagen volume and the myocyte area in the various groups $\left(R^{2}=0.73\right)$.

A positive correlation, $r=0.53, p=0.04$, was also seen between LVEDP and SGLS.

Systolic function. The LVEF was within normal limits in all patient groups, and there were no differences in LVMI, MFS at baseline and normalized by wall stress or LVESP measured by cardiac catheterization (Table 1). However, differences were seen in a preload-adjusted systolic function index $\left(+\mathrm{dP} / \mathrm{dt}_{\text {máx }}[\mathrm{mm} \mathrm{Hg} / \mathrm{s}]\right.$ normalized by LVEDP [mm Hg]) and in SGLS, thus, impairment of systolic function was more marked in patients with decompensated hypertrophy (Figs. 5, 6).

A positive correlation, $\mathrm{r}=0.71, \mathrm{p}=0.007$, was found between the hemodynamic index of

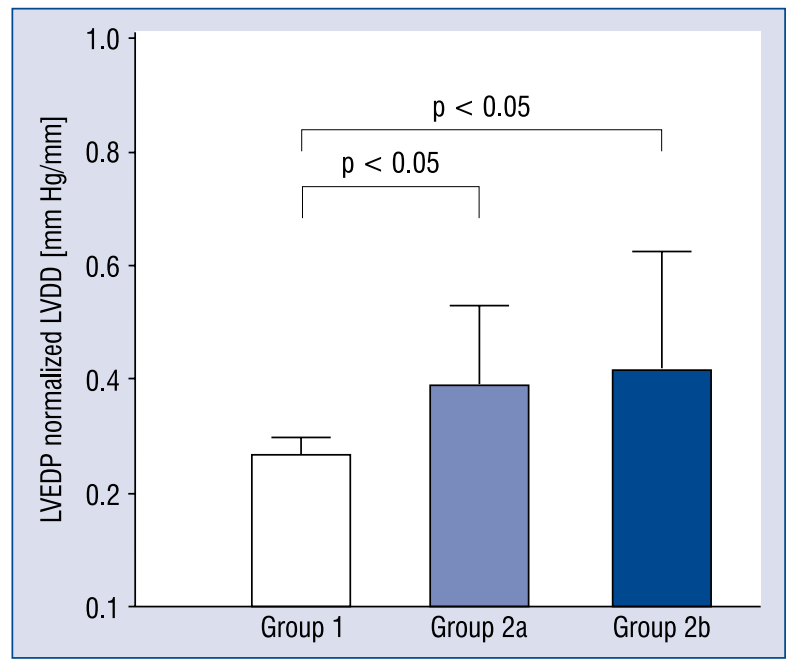

Figure 3. Graphic depicting differences in myocardial stiffness between groups; LVEDP — left ventricular enddiastolic pressure; LVDD - left ventricular diastolic diameter.

contractile status $\left(+\mathrm{dP} / \mathrm{dt}_{\text {máx }}[\mathrm{mm} \mathrm{Hg} / \mathrm{s}]\right.$ normalized by LVEDP $[\mathrm{mm} \mathrm{Hg}])$ and SGLS.

\section{Discussion}

This study shows that in patients with severe $\mathrm{AS}$ and preserved $\mathrm{LV}$ function, the adaptive mechanism of hypertrophy exhibits various stages of progression, expressed by changes in LV function and structure, which allow to identify groups of patients with different degrees of myocardial 


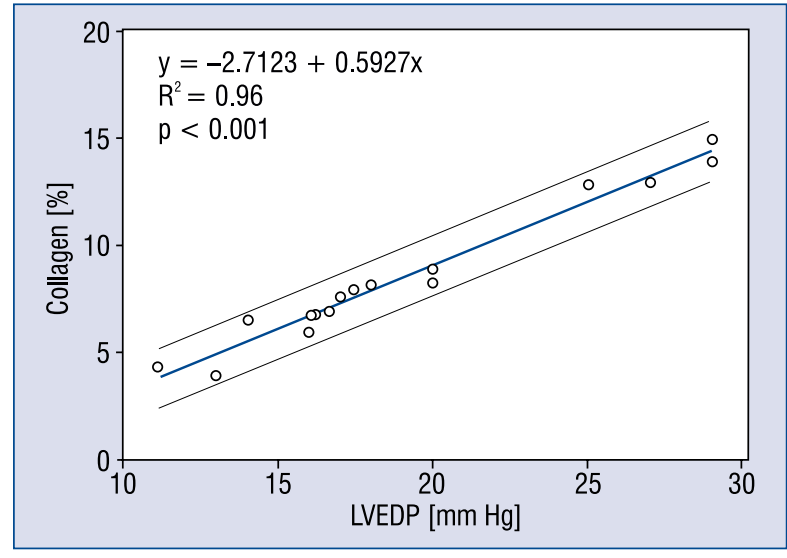

Figure 4. Linear regression of the relation between left ventricular end-diastolic pressure (LVEDP) in $\mathrm{mm} \mathrm{Hg}$ and collagen volume in percent (correlation coefficient $=0.96$, $\mathrm{p}<0.001$ ).

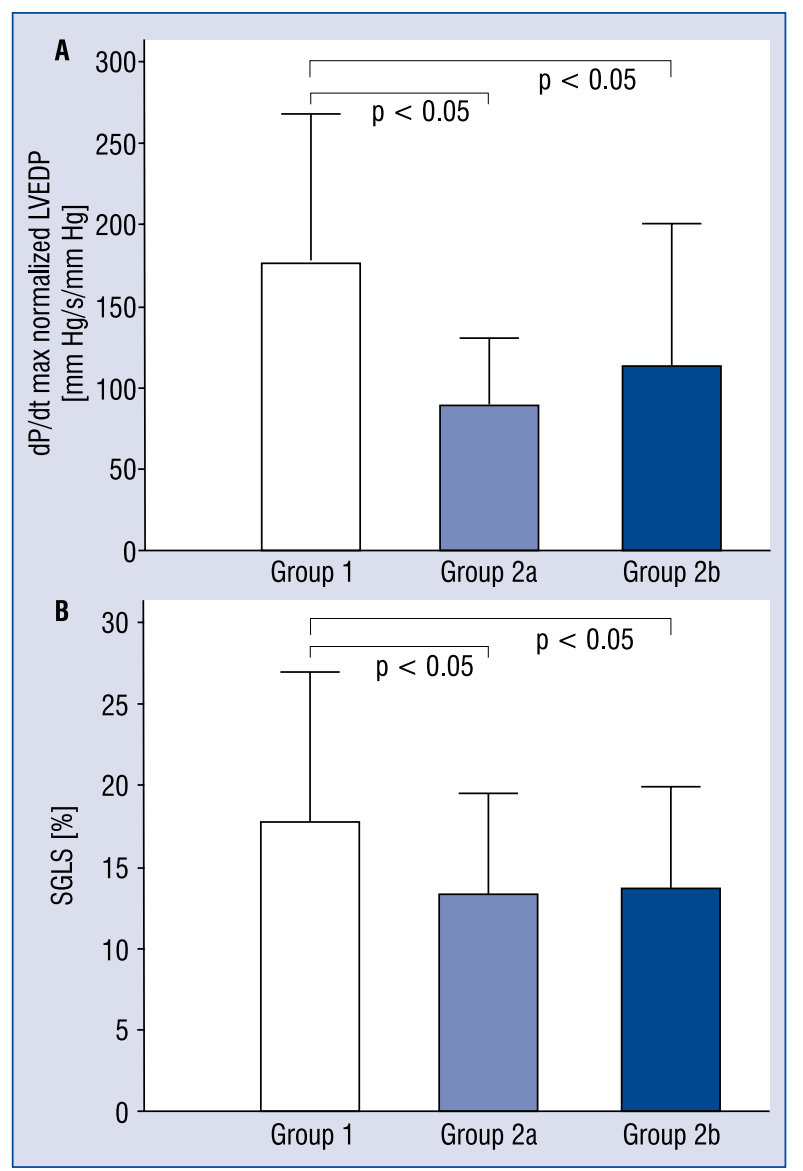

Figure 5. A. Analysis of $+\mathrm{dP}^{\mathrm{d}} / \mathrm{dt}_{\max }(\mathrm{mm} \mathrm{Hg} / \mathrm{s})$ normalized by left ventricular end-diastolic pressure (LVEDP), for the three groups. (*p $<0.05$ G1 vs. G2a and G2b); B. Left ventricular peak systolic global longitudinal 2-dimensional strain (SGLS), expressed as percent, for the three groups ( ${ }^{*} p<0.05$ G1 vs. G2a and G2b).

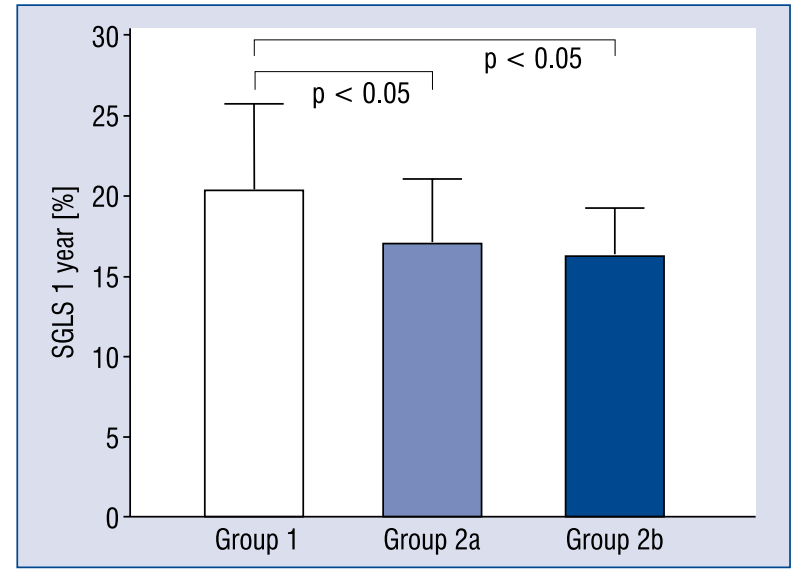

Figure 6. Left ventricular peak systolic global longitudinal 2-dimensional strain (SGLS) at 1 year post aortic valve replacement, expressed as percent, for groups G1, G2a and G2b (*p $<0.05$ G1 vs. G2a and G2b).

impairment. Early identification of such a myocardial impairment is important for decision making in valve disease in general, and particularly in AS. In the cases of increased wall stress, the myocardium develops ventricular hypertrophy as a compensatory response; such hypertrophy presents as quantitative and qualitative changes, increase in mass and changes in structure. In the present study, we considered the increase in collagen volume a qualitative change which occurred without differences in LVMI between groups. As Weber [25] stated: "It is not the quantity but rather the quality of myocardium that distinguishes hypertensive heart disease from adaptive hypertrophy of the athlete".

Decompensated groups exhibit greater impairment in structure characterized by a larger myocyte area and greater increase in collagen, with marked increase in myocardial stiffness. This impairment in diastolic function and its correlation with changes in structure was described in previous studies $[10,11]$, albeit in patients with less preserved $\mathrm{EF}$ than patients in our population, and without changes that allow to discriminate different groups with different stages of disease progression.

Although the increase in collagen volume of the compensated group is twofold the reference values for a normal population [26], it is much less than that found in the decompensated group. Increase in collagen is probably due to impairment in myocardial perfusion, secondary to the loss of coronary vasodilatory reserve and elevated wall stress, resulting in a predominant increase in subendocardial fibrosis as the disease progresses [27, 28]. 
Table 2. One-year echocardiography data.

\begin{tabular}{lcccc}
\hline Echocardiography at 1 year & Group 1 & Group 2a & Group 2b & P \\
\hline Ejection fraction [\%] & $62.75 \pm 13.81$ & 55 & $65 \pm 5.83$ & NS \\
End-systolic diameter [mm] & $27.5 \pm 2.38$ & 37 & $30.75 \pm 4.27$ & NS \\
End-diastolic diameter [mm] & $48.25 \pm 2.87$ & 61 & $48.75 \pm 3.77$ & NS \\
Myocardial mass index [g/m $\left.{ }^{2}\right]$ & $136.2 \pm 54.97$ & $158.66 \pm 24.36$ & $159.18 \pm 35.45$ & NS \\
Midwall fractional shortening [\%] & $17.63 \pm 1.42$ & $16.24 \pm 2.51$ & $14.62 \pm 1.35$ & 0.01 \\
Midwall fractional shortening/stress & $18.09 \pm 0.49$ & $18.29 \pm 0.22$ & $18.47 \pm 0.58$ & NS \\
\hline
\end{tabular}

1 - compensated hypertrophy; $2 \mathrm{a}$ - decompensated hypertrophy without coronary artery disease; $2 \mathrm{~b}-$ decompensated hypertrophy with coronary artery disease; NS - not significant

A special condition is that of patients with concomitant $\mathrm{CAD}$, in whom this mechanism adds on to those previously described.

All groups had impaired myocardial relaxation, as assessed by tau to $_{0}$; these changes seen in AS have been previously described in animal models [29], as well as in clinical studies [30], and are related (among other processes) to intracellular movement of calcium, thus it could be part of the mechanisms resulting in progressive failure of the hypertrophic myocardium [31].

Impairment of diastolic function in AS has been described as a prognostic factor, pre- and postoperatively, and some authors have suggested that its finding should be considered for early surgical intervention [32].

All patients in our study had a normal EF, a parameter that is used to express absence of failure of the global LV function; however, this index is subject to variations depending on the chamber's geometry, afterload and increase in mass [33-35], among other variables. Hence, EF is more precise to evaluate pump function than ventricular function, since it mainly expresses the radial function of the myocardium and is less affected by abnormalities of the subendocardium [36]. A more specific index of the assessment of function in the presence of hypertrophy, MFS [37], was normal in all group after normalizing by wall stress, probably because it was evaluated in patients with normal EF and an end-systolic stress which did not exceed $120 \mathrm{kdynes} / \mathrm{cm}^{2}$ (according to several studies, LVH should be considered inappropriate when values exceed this cutoff level) [18]; this could explain the failure of this index in detecting contractile function impairment in this study.

More sensitive parameters useful to assess contractile status are: $+\mathrm{dP} / \mathrm{dt}_{\max }$ [38] normalized by LVEDP, and SGLS, which allows to assess contractile properties of the myocardium [39] in various scenarios including AS [13], confirmed the impairment of systolic function, especially in decompensated groups, and could thus be useful tools to characterize subtle changes in ventricular function. Interestingly, a significant positive correlation was observed between both parameters assessing $\mathrm{LV}$ contractile status.

This myocardial impairment and structural changes initially and mainly involve the subendocardium [40], affecting longitudinal function, which is not well assessed by EF, but can be assessed by other indices that use M-mode and/or tissue Doppler imaging to analyze the lateral displacement of the mitral annulus [41].

Various authors have observed the association between strain impairment in patients with SSAS and its impact on increased risk and clinical events in the long-term follow-up [42].

Lancellotti et al. [43] studied patients with asymptomatic AS and saw that a SGLS $<15.9 \%$ was a prognostic marker of an adverse course. In our patients, the compensated group exhibited values above the cutoff point, whereas in the decompensated group with and without $\mathrm{CAD}$, values were $13.5 \%$ and $13.6 \%$, respectively. Other authors demonstrated that SGLS returned to normal values at 1 year post valve replacement [44]; in our study SGLS values returned to normal only in the compensated group (Table 2).

Correlation between the degree of fibrosis and changes in strain has been described by other authors [45] and by our group [14], as well as the improvement and/or normalization of strain values after aortic valve replacement [46]; however, we are not aware of any publication showing that in patients with preserved EF and MFS at baseline, two groups of patients could be distinguished by structure, function, baseline systo-diastolic function and a different strain pattern at 1 year. Evi- 
dence shows that changes in structure, particularly fibrosis, are an early finding in the progression of AS [47] and determine aspects of systolic, as well as diastolic function [48], which entail prognostic value and affect long-term survival following aortic valve replacement [49]. This adverse progression is related to myocardial failure which is probably an evolving process, as described by Meerson [50], and of which many aspects remain unknown. Early detection of myocardial impairment is clinically important, and detecting the various stages of progression of hypertrophy can be very useful for decision making.

The present study contributes information about the changes in cardiac structure and function in AS that may be useful for clinical management, and suggest a transition in the progression of ventricular hypertrophy to myocardial failure. Some of these changes remain abnormal at 1 year after valve replacement in the group of patients with decompensated hypertrophy.

\section{Limitations of the study}

Our results should be validated in a larger number of patients. The information found is only applicable to a symptomatic patient population. One-year follow-up does not provide information about the clinical course and LV function changes in the mid- and long-term follow-up.

\section{Conclusions}

Patients with SSAS and preserved EF who progress to decompensated LVH characterized by an increase in LVEDP, exhibit impairment in their myocardial structure and diastolic function expressed by changes in ventricular relaxation and myocardial stiffness, as well as in their systolic function expressed by hemodynamic and myocardial strain indices.

\section{Conflict of interest: None declared}

\section{References}

1. Kang D, Rim S, Yun S et al. Early surgery versus conventional treatment in asymptomatic very severe aortic stenosis. Circulation, 2010; 121: 1502-1509. doi: 10.1161/CIRCULATIONAHA. 109.909903.

2. Lancellotti P, Magne J, Donal E et al. Clinical outcome in asymptomatic severe aortic stenosis insights from the new proposed aortic stenosis grading classification. J Am Coll Cardiol, 2012; 59: 235-243. doi: 10.1016/j.jacc.2011.08.072.

3. Rosenhek R, Zilberszac R, Schemper M et al. Natural history of very severe aortic stenosis. Circulation, 2010; 121: 151-156. doi: 10.1161/CIR.0b013e3181cbbef1.
4. Bonow RO, Carabello BA, Kanu C et al. ACC/AHA 2006 guidelines for the management of patients with valvularheartdisease: A report of the American College of Cardiology/American Heart Association Task Force on Practice Guidelines (writing committee to revise the 1998 Guidelines for the Management of Patients with ValvularHeart Disease): Developed in collaboration with the Society of Cardiovascular Anesthesiologists: endorsed by the Society for Cardiovascular Angiography and Interventions and the Society of Thoracic Surgeons. Circulation, 2006; 114: e84-e231. doi: 10.1161/CIRCULATIONAHA.106.176857.

5. Vahanian A, Alfieri O, Andreotti F et al. Guidelines on the management of valvular heart disease (version 2012) The Joint Task Force on the Management of Valvular Heart Disease of the European Society of Cardiology (ESC) and the European Association for Cardio-Thoracic Surgery (EACTS). Eur Heart J, 2012; 33: 2451-2496. doi: 10.1093/eurheartj/ehs109.

6. Kennedy K, Nishimura, Holmes Jr, Bailey K. Natural history of moderate aortic stenosis. J Am Coll Cardiol, 1991; 17: 313-319.

7. Rosenhek R, Klaar U, Schemper M et al. Mild and moderate aortic stenosis. Natural history and risk stratification by echocardiography. Eur Heart J, 2004; 25: 199-205. doi: 10.1016/j. ehj.2003.12.002.

8. Krayenbuehl H, Hess O, Monrad E, Schneider J, Mall, Turina M. Left ventricular myocardial structure in aortic valve disease before, intermediate, and late after aortic valve replacement. Circulation, 1989; 79: 744-755. doi: 10.1161/01.CIR.79.4.

9. Beach J, Mihaljevic T, Svensson L et al. Coronary artery disease and outcomes of aortic valve replacement for severe aortic stenosis. J Am Coll Cardiol, 2013; 61: 837-848. doi: 10.1016/j. jacc.2012.10.049.

10. Hein S, Arnon E, Kostin S et al. Progression from compensated hypertrophy to failure in the pressure-overloaded human heart Structural deterioration and compensatory mechanisms. Circulation, 2003; 107: 984-991. doi: 10.1161/01. CIR.0000051865.66123.B7.

11. Villari B, Campbell S, Hess O et al. Influence of collagen network on left ventricular systolic and diastolic function in aortic valve disease. J Am Coll Cardiol, 1993; 22: 1477-1484.

12. Schultz O, Brala D, Bensch R et al. Aortic valve replacemente in asymtomatic and symptomatic patients with preserved left ventricular ejection fraction. J Heart Valve Dis, 2012; 21: 576-583.

13. Delgado V, Tops $\mathrm{L}$, vanBommel $\mathrm{R}$ et al. Strain analysis in patients with severe aortic stenosis and preserved left ventricular ejection fraction undergoing surgical valve replacement. Eur Heart J, 2009; 30: 3037-3047. doi: 10.1093/eurheartj/ehp351.

14. Hita A, Baratta S, Donato M et al. Study of the ventricular function and its correlation with morphometry in patients with severe symptomatic aortic stenosis. Eur Heart J, 2012; 33 (Abstract Supplement): 569, P3375.

15. Peterson K, Tsuji J, Johnson A, DiDonna J, LeWinter M. Diastolic left ventricular pressure-volume and stress-strain relations in patients with valvular aortic stenosis and left ventricular hypertrophy. Circulation, 1978; 58: 77-89. doi: 10.1161/01.CIR.58.1.77.

16. Schiller N, Shah P, Crawford M et al. Recommendations for quantitation of the left ventricleby two-dimensional echocardiography. American Society of Echocardiography Committee on Standards, Subcommittee on Quantitation of Two-Dimensional Echocardiograms. J Am SocEchocardiogr, 1989; 2: 358-367. doi: 10.1016/ S0894-7317(89)80014-8.

17. Shimizu G, Zile M, Blaustein A, Gaasch WH. Left ventricular chamber filling and midwall fiber lengthening in patients with left ventricular hypertrophy: Overestimation of fiber velocities by conventional midwall measurements. Circulation, 1985; 71: 266-72. doi: 10.1161/01.CIR.71.2.266.

18. Serneri G, Modesti P, Boddi M et al. Cardiac growth factors in human hypertrophy: Relations with myocardial contractility and wall stress. Circ Res, 1999; 85: 57-67. 
19. Lang R, Bierig M, Devereux R et al. Recommendations for Chamber Quantification: A Report from the American Society of Echocardiography's Guidelines and Standards Committee and the Chamber Quantification Writing Group, Developed in Conjunction with the European Association of Echocardiography, a Branch of the European Society of Cardiology. J Am Soc Echocardiogr, 2005; 18: 1440-1463. doi: 10.1016/j.echo.2005.10.005.

20. Korinek J, Wang J, Sengupta $P$ et al. Two-dimensional strain: A Doppler-independent ultrasound method for quantitation of regional deformation: Validation in vitro and in vivo. J Am Soc Echocardiogr, 2005; 18: 1247-1253. doi: 10.1016/j.echo.2005.03.024.

21. Langeland S, D'hooge J, Wouters PF et al. Experimental validation of a new ultrasound method for the simultaneous assessment of radial and longitudinal myocardial deformation independent of insonation angle. Circulation, 2005; 112: 2157-2162. doi: 10.1161/CIRCULATIONAHA.105.554006.

22. Perk G, Tunick PA, Kronzon I. Non-Doppler two-dimensional strain imaging byechocardiography: From technical considerations to clinical applications. J Am Soc Echocardiogr, 2007; 20: 234-243. doi: 10.1016/j.echo.2006.08.023.

23. Little WC. The left ventricular $+\mathrm{dP} / \mathrm{dtmax}$ end diastolic volume relation in closed-chest dogs. Circ Res, 1985; 56: 808. doi: 10.1161/01.RES.56.6.808.

24. Gonzalez E, Seropian I, Krieger M et al. Effect of early versus late AT 1 receptor blockade with losartan on postmyocardial infarction ventricular remodelingAm J Physiol Heart Circ Physiol, 2009; 297: H375-H386. doi: 10.1152/ajpheart.00498.2007.

25. Weber KT. Cardioreparation in hypertensive heart disease. Hypertension, 2001; 38 (Part 2): 588-5891.

26. Querejeta R, Nerea Varo S., Lopez B et al.Serum carboxy-terminal propeptide of procollagen type I is a marker of myocardial fibrosis in hypertensive heart disease Circulation, 2000; 101: 1729-1735. doi: 10.1161/01.CIR.101.14.1729.

27. Heymans S, Schroen B, Vermeersch P et al. Increased cardiac expression of tissue inhibitor of metalloproteinase- 1 and tissue inhibitor of metalloproteinase-2 is related to cardiac fibrosis and dysfunction in the chronic pressure-overloaded human heart. Circulation, 2005; 112: 1136-1144. doi: 10.1161/CIRCULATIONAHA.104.516963.

28. Villari B, Vassalli G, Betocchi S, Briguori C, Chiariello M, Hess OM. Normalization of left ventricular nonuniformity late after valve replacement for aortic stenosis. Am J Cardiol, 1996; 78: 66-71. doi: 10.1016/S0002-9149(96)00229-9.

29. Lecarpentier Y, Waldenström A, Clergue $\mathrm{M}$ et al. Major alterations in relaxation during cardiac hypertrophy induced by aortic stenosis in guinea pig. Circ Res, 1987; 61: 107-116. doi: 10.1161/01.RES.61.1.107.

30. Silberman G, Fan T, Liu H et al. Uncoupled cardiac nitric oxide synthase mediates diastolic dysfunction. Circulation, 2010; 121 : 519-528. doi: 10.1161/CIRCULATIONAHA.109.883777.

31. Zhang Y, Zhang M, Sears C et al. Reduced phospholamban phosphorylation is associated with impaired relaxation in left ventricular myocytes from neuronal NO synthase-deficient mice. Circ Res, 2008; 102: 242-224. doi: 10.1161/CIRCRESAHA.107.164798,

32. Lund $\mathrm{O}$, Flof $\mathrm{C}$, Jensenf $\mathrm{F}$ et al. Left ventricular systolic and diastolic function in aortic stenosis. Prognostic value after valve replacement and underlying mechanisms. Eur Heart J, 1997; 18: 1977-1987.

33. Aurigemma GP, Silver KH, Priest MA, Gaasch WH. Geometric changes allow normal ejection fraction despite depressed myocardial shortening in hypertensive left ventricular hypertrophy. J Am Coll Cardiol, 1995; 26: 195-202. doi: 10.1016/07351097(95)00153-Q.
34. Donal1 E., Bergerot $\mathrm{C}$, Thibault1 $\mathrm{H}$ et al. Influence of afterload on left ventricular radial and longitudinal systolic functions: A two-dimensional strain imaging study. Eur J Echocardiogr, 2009; 10: 914-921. doi: 10.1093/ejechocard/jep095.

35. Palmon LC, Reicheck N, Yeon SB et al. Intramural myocardial shortening in hypertensive left ventricular hypertrophy with normal pump function. Circulation, 1994; 89: 122-131. doi: 10.1161/01.CIR.89.1.122.

36. Maciver DH, Townsend M. A novel mechanism of heart failure with normal ejection fraction. Heart, 2008; 94: 446-449. doi: 10.1136/hrt.2006.114082.

37. Ballo P, Mondillo S, Guerrini F, Barbati R, Picchi A, Focardi M. Midwall mechanics in physiologic and hypertensive concentric hypertrophy. J Am Soc Echocardiogr, 2004; 17: 418-427. doi: 10.1016/j.echo.2004.01.011.

38. Katz. AM. Regulation of myocardial contractility 1958-1983: An odyssey. J Am Coll Cardiol, 1983; 1: 42-51. doi: 10.1016/S07351097(83)80009-6.

39. Weidemann F, Jamal F, Sutherland G et al. Myocardial function defined by strain rate and strain during alterations in inotropic states and heart rate. Am J Physiol Heart Circ Physiol, 2002; 283: H792-H799. doi: 10.1152/ajpheart.00025.2002.

40. Heymans S, Schroen B, Vermeersch P et al. Inhibitor of metalloproteinase-2 is related to cardiac fibrosis and dysfunction in the increased cardiac expression of tissue inhibitor of metalloproteinase- 1 and tissue chronic pressure-overloaded human heart. Circulation, 2005; 112: 1136-1144. doi: 10.1161/CIRCULATIONAHA.104.516963.

41. Takeda S, Rimington H, Smeeton N, Chambers J. Long axis excursion in aortic stenosis. Heart, 2001; 86: 52-56. doi: 10.1136/heart.86.1.52.

42. Lafitte S, Perlant M, Reant P et al. Impact of impaired myocardial deformations on exercise tolerance and prognosis in patients with asymptomatic aortic stenosis. Eur J Echocardiogr, 2009; 10: 414-419. doi: 10.1093/ejechocard/jen299.

43. Lancellotti P, Donal E, Magne J et al. Risk stratification in asymptomatic moderate to severe aortic stenosis: The importance of the valvular, arterial and ventricular interplay. Heart, 2010; 96: e1364-e1371. doi: 10.1136/hrt.2009.186486.

44. Rost C, Korder S, Wasmeier G et al. Sequential changes in myocardial function after valve replacement for aortic stenosis by speckle tracking echocardiography. Eur J Echocardiogr, 2010; 11: 584-589. doi: 10.1093/ejechocard/jeq017.

45. Weidemann F, Herrmann S, Störk S et al. Impact of myocardial fibrosis in patients with symptomatic severe aortic stenosis. Circulation, 2009; 120: 577-584. doi: 10.1161/CIRCULATIONAHA.108.847772.

46. Poulsen S, DMSci, Søgaard P et al. Recovery of left ventricular systolic longitudinal strain after valve replacement in aortic stenosis and relation to natriuretic peptides. J Am Soc Echocardiogr, 2007; 20: 877-884. doi: 10.1016/j.echo.2006.11.020.

47. Hein S, Arnon E, Kostin S et al. Progression from compensated hypertrophy to failure in the pressure-overloaded human heart structural deterioration and compensatory mechanisms. Circulation, 2003; 107: 984-991. doi: 10.1161/01. CIR.0000051865.66123.B7.

48. Weber K, Brilla C. Pathological hypertrophy and cardiac interstitium. Fibrosis and renin-angiotensin-aldosterone system. Circulation, 1991; 83: 1849-1865. doi: 10.1161/01.CIR.83.6.1849.

49. Milano A, Faggian G, Dodonov M, et al. Prognostic value of myocardial fibrosis in patients with severe aortic valve stenosis. J Thorac Cardiovasc Surg, 2012; 144: 830-837. doi: 10.1016/j. jtcvs.2011.11.024.

50. Meerson FZ. The failing heart. In: Katz AM eds. Adaptation and deadaptation. Raven, New York 1983. 